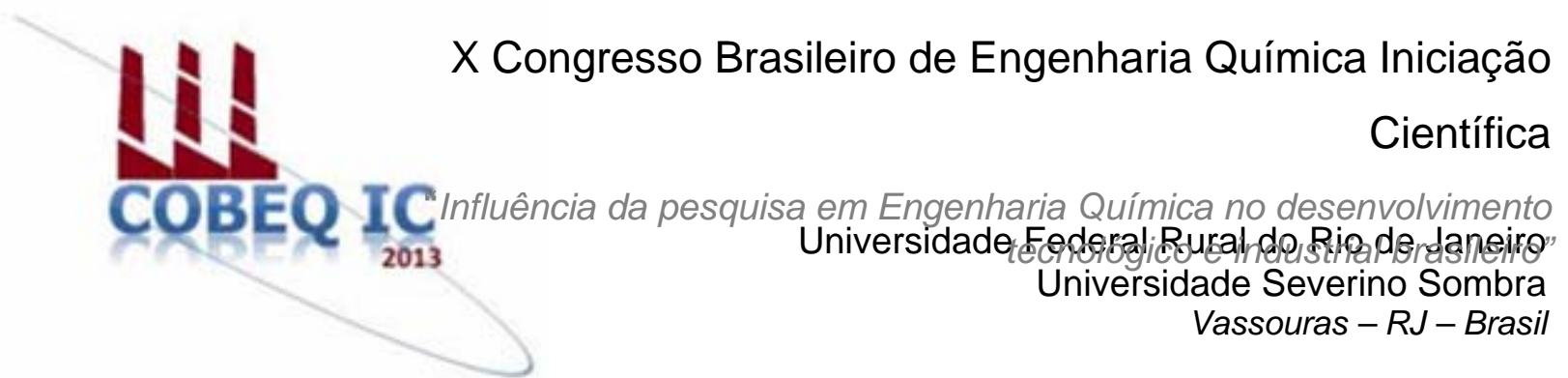

\title{
ESTUDO DE CONTROLADORES PI/ PPI PARA REGULAR A PRESSÃO ANULAR DE FUNDO
}

\author{
Priscila de Souza Breves Lima ${ }^{1}$, Willian Simões Cavalhiere ${ }^{1}$, Márcia Peixoto Vega ${ }^{2}$ \\ ${ }^{(1)}$ Bolsista de Iniciação Científica - PIBIC; \\ (2) Docente - DEQ/IT/UFRRJ \\ Departamento de Engenharia Química - Instituto de Tecnologia - UFRRJ, BR465, Km 7 - Seropédica, RJ - CEP \\ 23890-000 - e-mail: vega@ufrrj.br
}

\begin{abstract}
RESUMO - Em se tratando de perfuração de poços de petróleo, é importante o balanço de pressão entre o poço e o reservatório. Quando a pressão anular de fundo de poço é maior que a pressão da formação, ocorrerá invasão do fluido de perfuração através da rocha, que acarretará no comprometimento da produção do óleo. No entanto, se a pressão nos poros do reservatório for menor que a pressão no fundo do poço, ocorrerá um influxo dos fluidos do reservatório em direção ao poço. Uma das estratégias de controle da pressão anular de fundo é utilizar a modelagem de controle clássico (controlador PI), porém, uma nova forma de controle (controlador preditivo PI) foi proposta e analisada como tema central deste trabalho. Nesta estratégia de controle PPI, a ação integral é inibida durante o período de tempo igual ao tempo morto presente no processo, isto previne o windup da ação integral durante este período. Para efeitos de comparação do desempenho destes dois tipos de controladores, foram realizados testes utilizando como variável manipulada a abertura da válvula choke, fazendo uso primeiramente de um controlador PI e atrasos na aquisição dos dados da variável medida de 100 segundos e na variável manipulada de 100 segundos. Posteriormente, foram realizados os mesmos testes fazendo uso da abordagem PPI.
\end{abstract}

Palavras chave: perfuração de poços, controle com atraso, janela operacional.

\section{INTRODUÇÃO}

O início do processo de busca e exploração começou em torno de 1859 com a descoberta do primeiro poço de petróleo nos Estados Unidos - Pensilvânia. A primeira guerra mundial pôs então em evidência a importância estratégica do petróleo, através de sua utilização como material de guerra e pelo uso generalizado de seus derivados.

Hoje em dia é possível observar que o mercado mundial está direcionado quase que totalmente para o consumo de petróleo e seus derivados, desta forma este se tornou um recurso estratégico para todos os países. A indústria do petróleo é marcada por inúmeras particularidades, entre elas o fato de ser um recurso mineral não renovável e a principal fonte de energia consumida no mundo. Deste modo, por ser um material bem valioso para o mercado, a produção petrolífera é uma das atividades mais rendosas. Mas por outro lado, são necessários elevados investimentos para encontrar um poço de petróleo e iniciar a sua produção.

Uma das etapas mais custosas é a perfuração do poço, atualmente, o método mais utilizado nas perfurações de poços é o rotativo, que consiste em descer rotacionando uma coluna de perfuração com uma broca de aço em sua extremidade. A combinação do peso sobre a broca e da sua rotação sobre a formação causam o esmerilhamento da rocha. Nessa etapa, o fluido injetado passa por dentro 
da coluna de perfuração, depois pela broca e ascende pela região anular existente entre as paredes do poço e a coluna de perfuração. A lama de perfuração é responsável por exercer uma pressão hidrostática entre a coluna de perfuração e as paredes do poço. Essa pressão, conhecida como pressão anular de fundo, normalmente é mantida num valor maior que pressão de poros do reservatório, evitando assim influxos em direção ao poço, e menor que a pressão de fratura, para evitar uma invasão da lama, comprometendo a produção de óleo.

\section{REVISÃO DA LITERATURA}

O controle automático tem se tornado essencial em operações industriais para controle de pressão, temperatura, viscosidade, entre outros. Segundo Kwong (2010) um sistema de controle deve suprimir a influência de perturbações externas, assegurar a estabilidade do processo e otimizar o seu desempenho.

Um sistema de controle por realimentação é aquele que mantém uma relação prescrita entre a saída de um sistema e o estado desejado, comparando assim seus valores e utilizando a diferença como um meio de controle. A estabilidade é fundamental neste tipo de sistema, pois ela tende a corrigir erros e causar oscilações de amplitudes constantes ou variáveis (OGATA, 1982).

Dentre os modelos básicos de controle por realimentação ("feedback"), o mais utilizado é o controle proporcional integral (PI). Nesse tipo de controlador o sinal de atuação relacionado ao erro é dado pela Equação 1.

$$
C(t)=c_{s}+K_{c} E(t)+\frac{K_{c}}{\tau_{i}} \int_{0}^{t} E(t) d t
$$

\section{Onde :}

$m(t)$ - variável de saída;

$\mathrm{m}_{g}$ - variável de saída quanto $\mathrm{e}(\mathrm{t})=0$;

$\mathrm{K}_{\mathrm{c}}$ - ganho proporcional;

$\tau_{\mathrm{i}}$ - tempo integral e;

$e(t)-$ sinal de erro.
Para determinação dos parâmetros do controlador (Kc e $\tau \mathrm{i}$ ), é necessário identificarse o processo. Esses parâmetros poderão ser determinados usando-se dois métodos: o de curva de reação (ZIEGLER \& NICHOLS, 1942) e o de Sundaresan \& Krishnawany (1977), que determinam os parâmetros do sistema $(\mathrm{K}, \tau$ e td).

No método de Sundaresan \& Krisnawany dois valores de tempo são propostos em relação ao tempo de entrada em regime permanente ( $\mathrm{t} 1$ e $\mathrm{t} 2$ ), sendo eles $35,3 \%$ e $83,3 \%$ desse valor, respectivamente. Assim, os parâmetros são calculados pelas Equações 2 e 3 .

$$
\begin{gathered}
\tau=0,67\left(t_{2}-t_{1}\right) \\
t_{d}=1,3 t_{1}-0,29 t_{2}
\end{gathered}
$$

Uma vez determinados os parâmetros do sistema, os parâmetros do controlador podem ser determinados pelos métodos de Cohen-Coon (1953) e Ziegler-Nichols (1942) de acordo com as equações apresentadas nas Tabelas 1 e 2 .

Tabela 1. Equações de ajuste do método de Cohen-Coon (adaptado de OGATA, 1982)

\begin{tabular}{|c|c|c|}
\hline $\begin{array}{c}\text { Tipo de } \\
\text { Controlado } \\
\mathbf{r}\end{array}$ & $\mathbf{K}_{\mathbf{c}}$ & $\boldsymbol{\tau}_{\mathbf{i}}$ \\
\hline $\begin{array}{c}\text { Proporcion } \\
\text { al-Integral }\end{array}$ & $\frac{1}{K} \frac{\tau}{t_{d}}\left(0,9+\frac{t_{d}}{12 \tau}\right)$ & $t_{d} \frac{30+3 \frac{t_{d}}{\tau}}{9+20 \frac{t_{d}}{\tau}}$ \\
\hline
\end{tabular}

Tabela 2. Equações de ajuste do método de Ziegler-Nichols (adaptado de OGATA,1982)

\begin{tabular}{|c|c|c|}
\hline Tipo de Controlador & $\mathbf{K}_{\mathbf{c}}$ & $\boldsymbol{\tau}_{\mathbf{i}}$ \\
\hline PI & $\frac{0,9}{t_{d} S}$ & $3,33 t_{d}$ \\
\hline
\end{tabular}


Tore Hagglund produziu um novo tipo de controlador, chamado controlador preditivo PI (PPI), onde a ação integral é inibida durante um período igual ao atraso do modelo, impedindo assim, o windup da ação integral durante este intervalo de tempo. Além disso, Tore Hagglund estudou o desempenho de controladores PI e PPI, utilizando diferentes atrasos e constantes de tempo, chegando à conclusão que a implementação do controlador PPI apresentou resultados melhores toda vez que o atraso foi maior que a constante de tempo (HAGGLUND, 1992 apud COX, DANIEL\& LOWDO, 1997).

O método de Tore Hagglund também é caracterizado por um sistema de primeira ordem com atraso e é possível obter os parâmetros do controlador a partir dos valores de $T$ e $K_{p}$ conforme Tabela 3.

Tabela 3 - Relações para ajuste via método de Tore Hagglund (HAGGLUND, 1992).

\begin{tabular}{|c|c|c|}
\hline Controlador & $\boldsymbol{K}_{\boldsymbol{c}}$ & $\boldsymbol{T}_{\boldsymbol{i}}$ \\
\hline $\mathrm{PPI}$ & $\frac{1}{\mathrm{Kp}}$ & $T$ \\
& \\
\hline
\end{tabular}

Nesse tipo de teste, após sofrer a perturbação e durante um intervalo de tempo idêntico ao tempo morto, o controlador realiza apenas uma ação proporcional, sem somar o erro da ação integral. Após esse período, o controlador volta a implementar a ação proporcional-integral.

\section{MATERIAIS E MÉTODOS}

Os testes referentes a utilização do programa de perfuração inteligente foi realizado no laboratório de Escoamento de Fluidos Giulio Massarani instalado no DEQ/IT/UFRRJ.

Com o objetivo de verificar se a planta apresenta alguma não linearidade foram implementados, na unidade de perfuração, testes degrau positivo/negativo, no índice de abertura da válvula de choke, com magnitude de $\pm 35 \%$, para diferentes vazões. Em seguida verificou-se o comportamento da unidade quando da implementação de diferentes perturbações na abertura da válvula de choke
(95-25\%; 95-35 \%; 95-55 \%). O objetivo principal é avaliar o comportamento da variável de saída (pressão) em toda a faixa operacional. Após a identificação do sistema, via método de Sundaresan e Krishnaswamy, calculou-se os parâmetros do controlador PI e PPI através dos métodos de Ziegler-Nichols, Cohen-Coon e Hagglund, T., e em seguida, esses parâmetros foram implementados em testes de controle servo (mudança de set point de pressão em degrau), para diferentes níveis de frequência e com atrasos na medição das variáveis do processo e na transmissão dos valores das variáveis medidas.

\section{RESULTADOS}

Observa-se que a magnitude e forma da resposta ao degrau positivo/negativo foram diferentes, evidenciando que o processo apresenta alguma não linearidade e indicando que o uso de uma estratégia de controle clássico (PI, por exemplo) pode apresentar desempenho insatisfatório, podendo ser necessário efetuar a sintonia do controlador para diferentes níveis operacionais ou partir para uma estratégia de controle não linear (Figura 1).

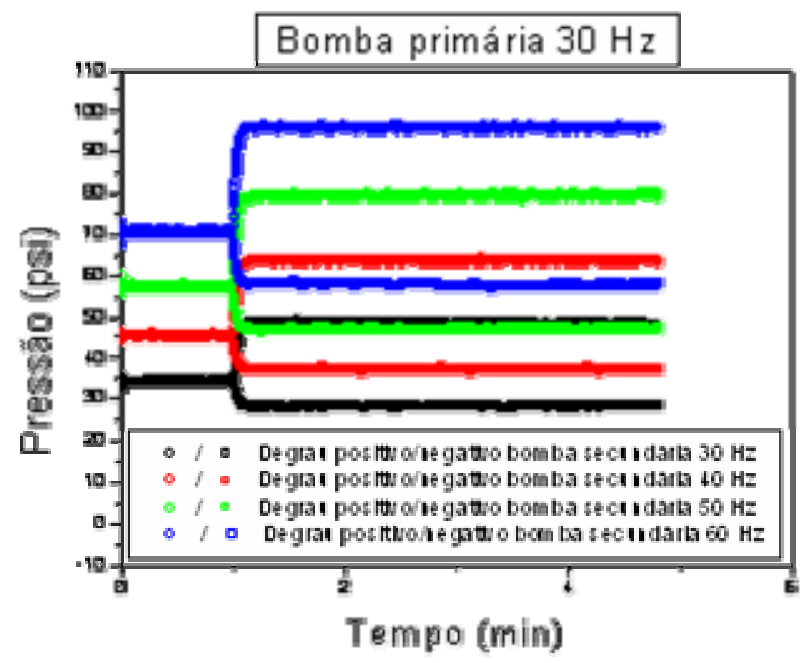

Figura 1- Testes degrau positivo/negativo para a frequência de $30 \mathrm{~Hz}$ na bomba primária.

A Figura 2 ilustra o comportamento da unidade quando da implementação de diferentes perturbações na abertura da válvula de choke (95-25\%; 95-35\%; 95-55\%). 


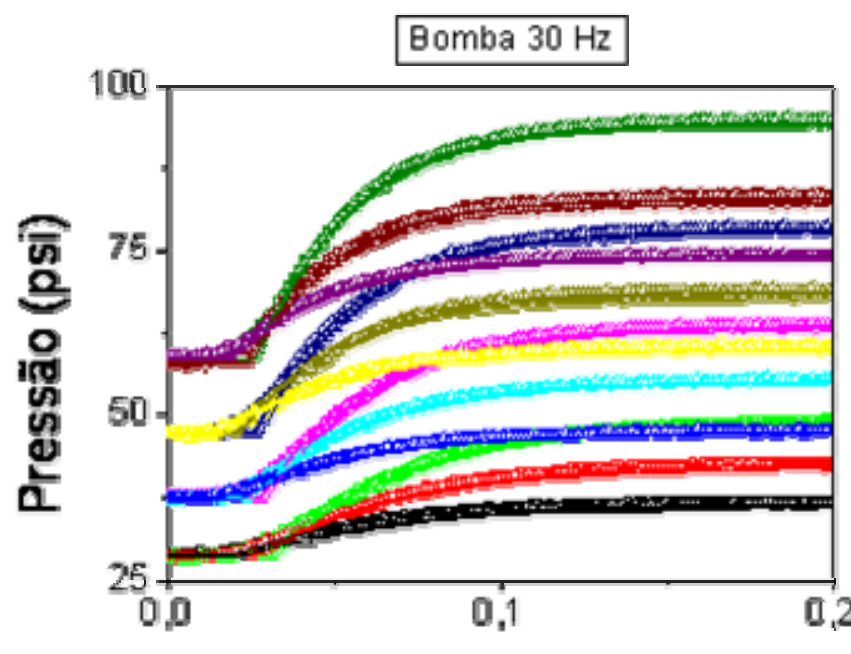

Figura 2- Curva de reação para a frequência de $40 \mathrm{~Hz}$ na bomba primária.

Observa-se que o tempo morto cresce ligeiramente à medida que as magnitudes das perturbações degrau na abertura da válvula crescem, indicando que este atraso é inerente ao tempo de resposta do equipamento (válvula). Verifica-se ainda que à medida que o nível de pressão aumenta, seja pelo fechamento da válvula ou pelo aumento da vazão de operação, o sistema responde mais rápido (a constante de tempo do sistema é menor), isto é, uma modificação na entrada (abertura da válvula) produz um rápido efeito na saída do sistema (pressão).

Durante a perfuração de poços de petróleo, dados como pressão são enviados através do fluido sendo bombeado e, portanto, não são recebidos em tempo real. Este atraso ou tempo morto faz com que a ação de controle na variável manipulada seja implementada com base em erros passados, fato que complica o desempenho da malha fechada.

A Figura 3 ilustra a implementação de testes de controle feedback PI/PPI servo rastreando os níveis desejados de pressão localizados acima da pressão de poros e abaixo da pressão de fratura. Foram utilizados atrasos de 100 s para a variável monitorada (pressão) e $100 \mathrm{~s}$ para a variável manipulada (choke).

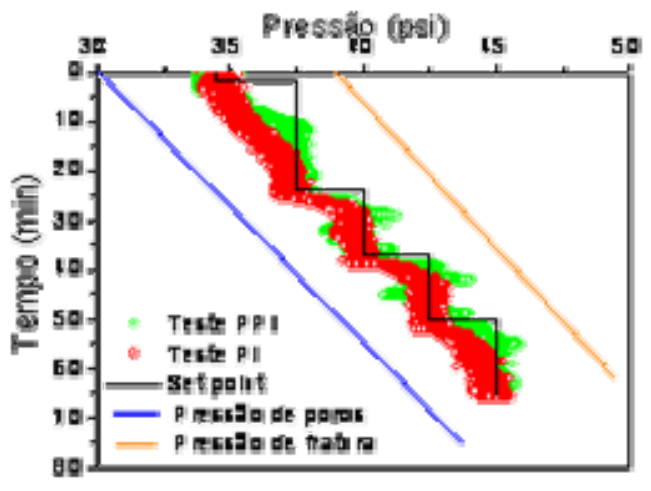

Figura 3- Comparação entre testes servo PI e PPI com janela operacional (Bomba de água

$30 \mathrm{~Hz}$ e bomba de lama $30 \mathrm{~Hz}$ ).

Como não foi possível acessar os reais valores da pressão de poros e de fratura, os valores utilizados para estas pressões no gráfico são apenas ilustrativos.

Pelo fato de no controlador preditivo PI (PPI), a ação integral ser inibida até um período igual ao atraso do modelo, os testes experimentais chegaram mais rápido ao valor final demonstrando um desempenho superior ao controlador feedback PI em malha fechada .

\section{CONCLUSÕES}

Foram avaliadas as estruturas PI versus PPI para unidade de perfuração de poços de petróleo, cuja característica marcante é apresentar atrasos nos sensores e atuadores. O controlador PPI apresentou maior velocidade de resposta e melhor desempenho em malha fechada quando comparado ao controlador PI. 


\section{REFERÊNCIAS}

COHEN, G.H. \& COON, G.A.. Theoretical Considerations of Retarded Control. Transactions of the ASME, jul. 1953.

COX, C.S., DANIEL,P.R.\&LOWDO, A. Quicktune: a reliable automatic strategy for determining pi and ppi controller parameters using a FOPT model.Control Eng. Practice, Vol. 5, No.10, pp. 1463-1472, 1997.

OGATA, K. Engenharia de Controle Moderno. Editora Prentice/Hall do Brasil. 4a Edição. 1982.

SUNDARESAN,K. R. \& KRISHNASWAMY P. R. Estimation of time delay time constant parameters in time, frequency, and Laplace domains Can J. Chem. Eng, 1977.

ZIEGLER, J.B. \& NICHOLS, N.B. Optimum settings for automatic controllers. ASME Transactions, Vol. 64, 1942.

\section{AGRADECIMENTOS}

FINEP/PETROBRAS 